\section{MS15-P10 A kaleidoscope of hypercoordinated alkali metal imidazolates: single source precursors for hybrid borohydrides}

Damir A. Safin ${ }^{1}$, Koen Robeyns ${ }^{1}$, Nikolay A. Tumanov ${ }^{1}$, Voraksmy Ban ${ }^{1}$, Yaroslav Filinchuk*1

1. Institute of Condensed Matter and Nanosciences, Université catholique de Louvain, Place L. Pasteur 1, 1348 Louvain-la-Neuve, Belgium

email: damir.a.safin@gmail.com

Zeolitic imidazolate frameworks (ZIFs) are an outstanding class of MOFs, constructed from tetrahedrally configured transition metal cations linked through bridging imidazolate (Im) spacers. ZIFs are structurally isiomorphic to zeolites since the metal-Im-metal angle is similar to the $\mathrm{Si}-\mathrm{O}-\mathrm{Si}$ angle $\left(145^{\circ}\right)$ in the latter compounds. This feature was exploited to produce a huge variety of porous transition metal-based ZIFs. Our initial goal was to combine Im and alkali metal borohydrides in the same structure with the formation of porous borohydride compounds, where Im serves as a structural unit of the framework, while borohydride anions provide with functionality. Surprisingly, we found that alkali metal-based imidazolates are not structurally characterized so far. This might be explained both by their general unavailability and by efficient hydrolysis. Thus, the coordination chemistry of Im towards alkali metal cations $\left(\mathrm{Li}^{+}, \mathrm{Na}^{+}\right.$ and $\mathrm{K}^{+}$) remains a challenge. With this in mind and inspired by the storage properties of ZIFs, we have turned our attention to Im-based coordination compounds with $\mathrm{Li}^{+}, \mathrm{Na}^{+}$and $\mathrm{K}^{+}$as well as determination of their structures.

Homoleptic 3D MOF-like structures $[\mathrm{Na}(\mathrm{Im})]_{n}$ and $[\mathrm{K}(\mathrm{Im})]_{n}$ (Figure 1 ) were obtained by the direct reaction of the corresponding hydroxide and $\operatorname{ImH}$, while $[\mathrm{Li}(\operatorname{Im})]_{n}$ was produced using a stepwise synthetic approach through the formation of heteroleptic compounds $[\mathrm{Li}(\operatorname{ImH})(\operatorname{Im})]_{n}$ and $[\mathrm{Li}(\mathrm{DMSO})(\mathrm{Im})]_{n}$ each exhibiting a $2 \mathrm{D}$ sheet-like structure. Using an equimolar mixture of two or three alkali metal hydroxides yielded heteronuclear homoleptic 3D MOF-like structures $\left[\mathrm{LiK}(\mathrm{Im})_{2}\right]_{n}, \quad\left[\mathrm{NaK}(\mathrm{Im})_{2}\right]_{n}, \quad\left[\mathrm{Na}_{2} \mathrm{~K}(\mathrm{Im})_{3}\right]_{n} \quad$ and $\left[\mathrm{Li}_{0.89} \mathrm{Na}_{1.12} \mathrm{~K}(\mathrm{Im})_{3}\right]_{\eta}$ or ${ }^{\prime}$ a heteroleptic ${ }^{2}$ complex $\left[\mathrm{Li}_{184} \mathrm{Na}_{1.16}(\mathrm{ImH})_{2}^{3}(\mathrm{Im})_{3}\right]_{1}$ constructed from $2 \mathrm{D}$ sheets. Thus, the presence of the parent ligand $\operatorname{ImH}$ in the structure of reported compounds decreases the dimensionality of the framework from $3 \mathrm{D}$ to $2 \mathrm{D}$. The coordinative demand of the metal cation in is increasing with an increase of the ionic radii. The $\mathrm{Li}^{+}$cation exhibits a strong propensity to form heteroleptic structures, while the $\mathrm{K}^{+}$cation allows to coordinate the Im ring through the $\pi$-system. While the alkali metal cations found in the CSD show preferred coordination to the nitrogen atom in the plane of Im, the alkali metal sites in this work are distributed over a larger range from 0 to $90^{\circ}$, and show even a wider distribution than for non-alkali metal cations found in the CSD (Figure 1).

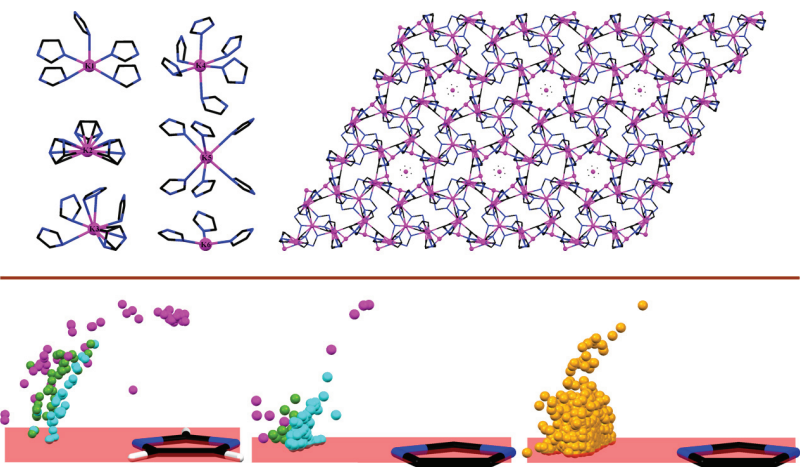

Figure 1. Coordination of the metal cations (top left) and packing (top right) in $[\mathrm{K}(\mathrm{Im})]_{\text {. }}$. Distribution of the metal sites around Im for the structures reported herein (bottom left), for the structures of substituted Im with alkali (bottom middle) and with other metal cations (bottom right).

Keywords: alkali metal cations, crystal structure, imidazole, metal organic frameworks, zeolitic imidazolate frameworks 